\title{
ATENCIÓN EN CENTROS ASISTENCIALES PARA LA MUJER VÍCTIMA DE VIOLENCIA DE GÉNERO DE COIRÓS (GALICIA) Y CARTAGENA DE INDIAS: UN ANÁLISIS COMPARADO
}

\section{ATTENTION IN HEALTHCARE CENTERS FOR WOMEN VICTIMS OF GENDER VIOLENCE OF COIROS (GALICIA) AND CARTAGENA DE INDIAS: A COMPARATIVE ANALYSIS}

\author{
Pedro Vázquez-Miraz ${ }^{1}$. \\ Universidad Tecnológica de Bolívar - Colombia \\ pvasquez@utb.edu.co \\ Angélica Quintana². \\ Universidad Tecnológica de Bolívar - Colombia \\ angelicaquintanadeavila@gmail.com \\ Begoña Riveira3. \\ Ayuntamiento de Coirós - España \\ begona.riveira@coiros.es
}

Resumen: En el presente estudio descriptivo-comparativo se analizaron los servicios prestados por dos centros asistenciales a mujeres víctimas de maltrato de España [Centro de Información a la Mujer (CIM) del municipio gallego de Coirós, de potestad pública ( $\mathrm{N}=$ 63)] y Colombia [Centro de Orientación a la Mujer (COM) situado en la ciudad de Cartagena de Indias, de responsabilidad privada $(\mathrm{N}=43)$ ] con el perfil de las usuarias que acudían a estos espacios de atención gratuita. Por medio de encuestas diseñadas especialmente para este estudio, y modificadas para cada entidad específica se ha pretendido analizar los niveles de satisfacción y conocimiento de dos servicios atencionales a víctimas de la violencia de género de dos países con tasas de feminicidios muy dispares e identificar las características personales de las usuarias de cada ente. Se identificó que los niveles de satisfacción por los servicios prestados y el desconocimiento previo de la utilidad de ambos centros fue muy elevada, observándose diferencias sociodemográficas respecto el perfil de la mujer maltratada que acudía a cada entidad.

Palabras clave: género, protección, satisfacción, sexismo, violencia.

Abstract: This descriptive-comparative study analysed the services provided by two
assistance centres for women victims of abuse in Spain [Centro de Información a la Mujer
(CIM) in the Galician municipality of Coirós, a public centre ( $\mathrm{N}=63)$ ] and Colombia
[Centro de Orientación a la Mujer (COM) located in the city of Cartagena de Indias, a
$\begin{array}{ll}\text { Orcid }^{1}: 0000-0002-5801-1728 & \text { Recibido: } 10.05 .2021 \\ \text { Orcid }^{2}: 0000-0002-2867-5064 & \text { Aceptado: } 16.12 .2021 \\ \text { Orcid }^{3}: 0000-0001-9538-4999 & \end{array}$ 
private centre $(N=43)$ ] with the profile of the users who attended these free assistance centres. By means of surveys designed especially for this study, and modified for each specific entity, the aim has been to analyse the levels of satisfaction and knowledge of two care services for victims of gender-based violence in two countries with very different rates of feminicide and to identify the personal characteristics of the users of each institution. It was identified that the levels of satisfaction with the services provided and the previous lack of knowledge of the usefulness of both centres was very high, with socio-demographic differences being observed with respect to the profile of the abused woman who attended each entity.

Keywords: gender, protection, satisfaction, sexism, violence.

\section{Introducción}

La problemática social de la violencia de género se ha tornado en los tiempos actuales como un núcleo de reflexión, crítica y atención especializada desde distintas áreas del saber humano (Sahagún y Arias, 2018), particularmente en los ámbitos de la psicología y el derecho. A nivel legal, se ha puesto de relieve el modo en que la igualdad entre hombres y mujeres se aplica de forma paradójica, existiendo en muchas ocasiones una igualdad en el ámbito teórico mientras que en la realidad la disparidad es lo que impera. Niño y Núñez (2009) coinciden plenamente con lo anterior y asumen la importancia que toman los específicos marcos jurídicos de cada país al impactar plenamente en la creación de un aparataje legal que busca prevenir las distintas manifestaciones de violencia contra la mujer, así como asistirlas en los casos en los que ya han ocurrido.

Este aspecto no es algo baladí, pues las nocivas consecuencias de las agresiones contra las mujeres "resultado de un daño físico, sexual o psicológico (...), tanto si se producen en la vida pública como en la privada" (OMS, 1998, p.3), requieren intervenciones psicosociales de carácter protector hacia las víctimas de distinta clase como los relacionados con la salud (física y/o mental), el apoyo jurídico o el apoyo económico y educativo entre otros (Vázquez-Miraz, 2017) puesto que "no existe una sola causa que determine la violencia del hombre contra la mujer" (López, 2005, p. 37). Por ello es fundamental que el papel de los centros asistenciales para mujeres que han sufrido la violencia de género tenga una mayor visibilidad y reconocimiento legal. El estudiar la satisfacción de las personas que reciben este tipo de prestaciones en diferentes contextos lo consideraríamos como una forma de lograr este propósito.

La Declaración sobre la Eliminación de la Violencia contra la Mujer de la Organización de las Naciones Unidas del año 1993 es uno de los primeros informes internacionales que abarca en su seno la especificidad de la agresión en contra de la mujer (Yugueros, 2014).

IQUAL. REVISTA DE GÉNERO E IGUALDAD, 2022, 5, 79-97 ISSN. 2603-851X DOI. http://dx.doi.org/10.6018/iqual.479851 
El documento proporciona una definición precisa de esta forma de violencia ${ }^{1}$ además de señalar, de forma genérica, la existencia de características sociales que favorecen este tipo de comportamientos. Así pues, las víctimas sufrirían esta violencia por su género (Bernal, 2011) y en base a su clase social, su etnia, su religión, su orientación sexual o edad también podrían padecer agresiones de corte físico, psicológico, sexual, patrimonial y económico en mayor proporción que otras féminas (Munévar y Mena, 2009); debiéndose recordar que estos actos también afectan a la educación recibida de los menores en los hogares que normalizan estos actos (Vázquez-Miraz, 2017).

El fundamentar este artículo científico desde el específico término de violencia de género requiere indicar de manera explícita que otros constructos similares, tales como la violencia contra la pareja ${ }^{2}$ o la violencia machista, afectan tanto a hombres como mujeres debido a aspectos personales de las víctimas como la orientación sexual, edad o estrato socioeconómico, entre otros factores (Gámez-Guadix et al., 2018). Un relevante debate teórico, pues como afirma Yugueros (2014) en este específico campo de trabajo es fundamental delimitar y nombrar de manera adecuada los conceptos teóricos, los métodos de intervención social y los sujetos participantes. Así pues, el equipo investigador del presente documento asumió al constructo de la violencia de género como un sinónimo de la violencia contra la mujer ejercida por el varón.

De este modo podemos indicar que la violencia de género se edifica sobre una configuración particular de nuestros imaginarios y cosmovisiones sociales respecto a los roles sexuales (Bem, 1974), puesto que "la violencia contra las mujeres es una expresión del poder y dominio de hombres sobre mujeres, fruto de la estructura social patriarcal que asigna roles de desigual valor a hombres y mujeres, y que se traducen en determinados estereotipos" (Romero, 2010, p. 191). En este sentido, y como indicaba Beauvoir (1949), la mujer en su condición de feminidad no nace siendo tal y sí es resultado de una dinámica social específica; pues más allá del específico condicionamiento orgánico del sujeto humano se desarrolla un constructo sociocultural de comportamientos y conductas femeninos. En palabras de esta misma autora:

\footnotetext{
1 "Todo acto de violencia basado en la pertenencia al sexo femenino, que tenga o pueda tener como resultado un daño o sufrimiento físico, sexual o psicológico para la mujer, así como las amenazas de tales actos, la coacción o la privación arbitraria de la libertad, tanto si se produce en la vida pública como en la vida privada" (Yugueros, 2014, p. 148).

${ }^{2}$ Concepto definido como "todo acto de violencia física y psicológica, incluidas las agresiones a la libertad sexual, las amenazas, las coacciones o la privación arbitraria de la libertad, que se ejerce sobre las mujeres por parte de quienes sean o hayan sido sus cónyuges o de quienes estén o hayan estado ligados en ella por relaciones similares de afectividad, aun sin convivencia" (Ley Orgánica 1 de España, 2004, p. 10) y entendido como "un fenómeno social que se ha presentado históricamente, el cual surge bajo estructuras de desigualdad entre el género masculino y femenino" (Moreno et al., 2019, p. 29).

IQUAL. REVISTA DE GÉNERO E IGUALDAD, 2022, 5, 79-97

ISSN. 2603-851X

DOI. http://dx.doi.org/10.6018/iqual. 479851
} 
No se nace mujer: se llega a serlo. Ningún destino biológico, psíquico o económico define la figura que reviste en el seno de la sociedad la hembra humana; es el conjunto de la civilización el que elabora ese producto intermedio entre el macho y el castrado al que se califica de femenino (Beauvoir, 1949, p. 87).

La consecuencia más importante que se deriva de naturalizar este conjunto de prácticas sociales en función del sexo de los individuos es que se pone en situación de desigualdad a la mujer respecto al hombre; la comprendida como constitución patriarcal de la sociedad. Un manido concepto entendido como el modelo de sociedad que considera a los varones como los sujetos que deben ocupar predominantemente puestos de mayor poder en las empresas en la política y por ende los que deben ser la autoridad y la fuerza dentro del hogar (Fernández-Ruiz, 2019).

Para paliar esta situación se han creado múltiples organizaciones y entidades con el fin de brindar apoyo a estas víctimas, siendo el primer centro bien documentado históricamente para la protección de este tipo de mujeres el creado en la ciudad de Hounslow (Reino Unido) en el año 1971. Un espacio donde se ofrecía refugio a mujeres maltratadas en el ámbito doméstico, abriéndose posteriormente similares entes en distintos países y regiones 3 (ONU, 2012). Siendo relevante el comparar el modo de trabajar de los servicios atencionales hacia las mujeres víctimas de violencia de género de diferentes contextos, como por ejemplo España y Colombia, país este último con unas mayores tasas de feminicidio (Figura 1 ) que puede ver en el anterior país europeo un modelo a seguir en relación con este tipo de actuaciones paliativas.

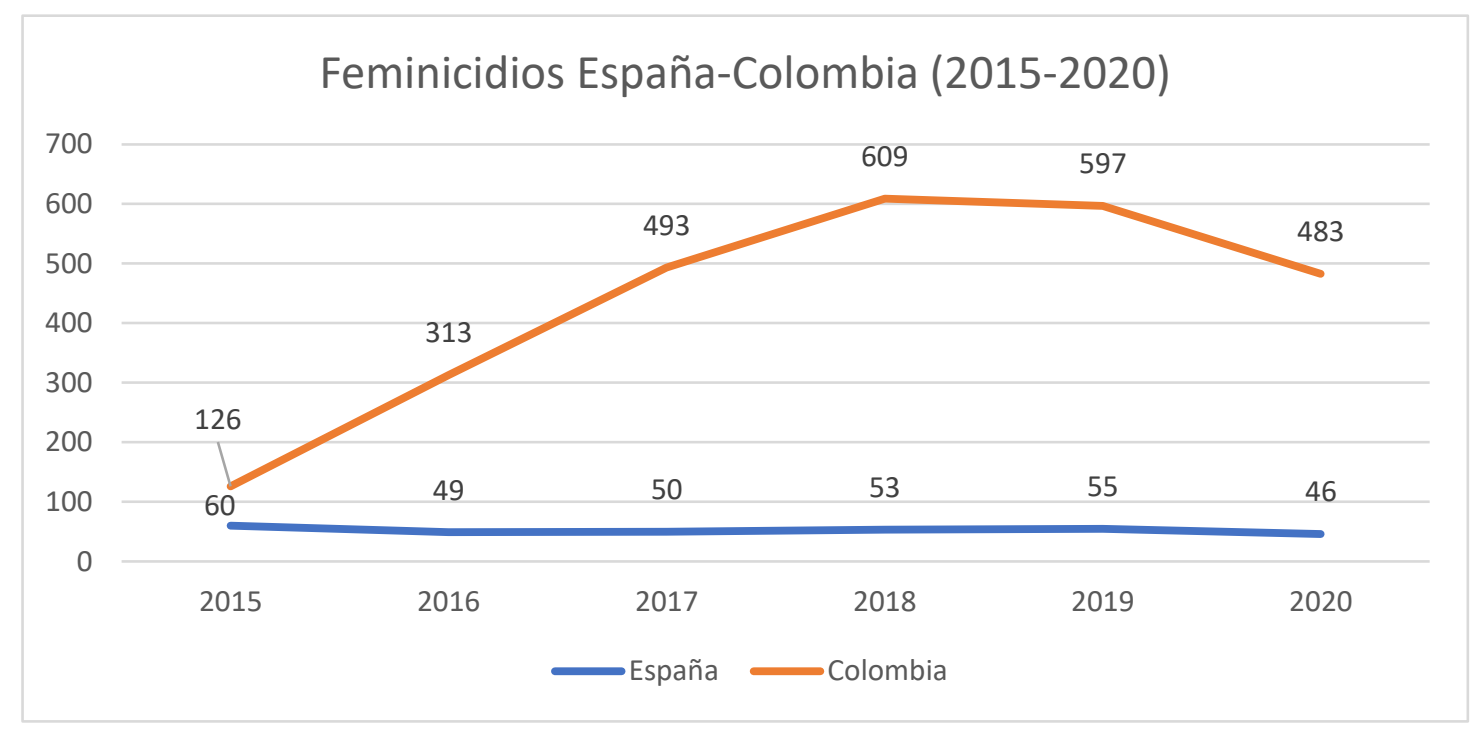

3 Estos servicios de refugio atendían lesiones físicas, aspectos emocionales relacionados con rupturas sentimentales, necesidades de servicios jurídicos, sociales y médicos y las demandas de los menores que llegaban acompañando a su madre (ONU, 2012).

IQUAL. REVISTA DE GÉNERO E IGUALDAD, 2022, 5, 79-97 ISSN. 2603-851X DOI. http://dx.doi.org/10.6018/iqual.479851 
Fuente: Ministerio de Igualdad de España (2021) y Ministerio de Tecnologías de la Información y las Comunicaciones de Colombia (2021)

Vistas las anteriores cifras, puede plantearse la idea de trabajo de que las mujeres españolas y colombianas que sufren malos tratos tendrían opiniones dispares de satisfacción respecto las instituciones que las intentan ayudar. Más si comparamos regiones tan específicas como Galicia (España) y Bolívar (Colombia). A modo de ejemplo, mientras que en la comunidad gallega (población de 2.700 .000 personas) en el año 2018 fallecían tres (3) mujeres a manos de sus (ex)parejas, siendo el pico más alto del ciclo histórico el de ocho (8) feminicidios en el 2015 (Ministerio de Presidencia de España,

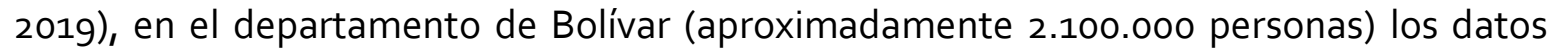
de fallecidas se aproximan más a los de toda España que a los de Galicia (Ministerio de Tecnologías de la Información y las Comunicaciones de Colombia, 2021).

Esta situación puede deberse a numerosos factores socio-económicos tales como la mayor carencia de recursos económicos o la gran aceptación social que tiene el comportamiento y el pensamiento machista en Colombia respecto al país ibérico, muy en particular en la ciudad de Cartagena de Indias (Zakzuk-López y Vázquez-Miraz, 2021). Mientras que el estudio de variables culturales asociado a conductas y pensamientos de mujeres que solicitan ayuda a centros atencionales de Galicia fue estudiado por San Martín (2012), las investigaciones transculturales entre América Latina y España respecto al funcionamiento de este tipo de espacios no son muy comunes, pudiéndose destacar los trabajos en España de González y Garrido (2015) o Rojas-Loria et al. (2015) como principales antecedentes. Es por ello por lo que nuestro aporte más novedoso sería el de querer comparar los niveles de satisfacción de víctimas de dos centros de atención a víctimas de violencia de género de dos regiones geográficas dispares, entendiéndose este concepto como "el grado de experiencia individual comparada con las expectativas" (Giese y Cote, 2000 citados en González y Garrido, 2015); ya que es asumido por la ciudadanía de manera general que los niveles de satisfacción en las usuarias de estos servicios son siempre positivos.

Aunque las autoridades del Ministerio de Salud de la República de Colombia (1999) ya señalaban a finales del siglo XX que la violencia contra la mujer era la expresión más clara de la relación desigual que se tiene entre distintos géneros, en el seno de las sociedades patriarcales; las cifras de muertes en este país sudamericano es una autentica lacra que no tiene comparación alguna con las (igualmente) trágicas cifras de España. Es por ello, desde el contexto del equipo investigador, por lo que se hace relevante el analizar los centros asistenciales de diferentes naciones para las mujeres víctimas de violencia de género que sean de similar cultura, identificar los niveles de satisfacción de las usuarias de estos servicios y los patrones identificativos de las personas que acuden a este tipo de 
instituciones para así comprender mejor el origen de la violencia de género, además de detectar posibles carencias o aspectos de mejora en este tipo de corporaciones.

\section{Marco jurídico de España y Colombia respecto la violencia de género}

Como afirma Yugueros (2021) el principal papel de los servicios de atención a las víctimas de género es el de informar a las personas que asisten a este tipo de centros de los derechos que éstas tienen como sujetos que padecen las agresiones machistas y así lograr una mayor autonomía, independencia y empoderamiento en sus vidas cotidianas. Por ello se requiere que los/as profesionales que trabajen en estos organismos tengan además de una formación específica en estudios de género, conozcan las disposiciones legales asociadas a esta particular problemática para así lograr un efectivo asesoramiento en los distintos campos en los que se trabaje, como las áreas psicológica, jurídica o de igualdad, etc.

A continuación, se presentan los primeros desarrollos normativos jurídicos de España y Colombia en relación con la violencia de género de principios del siglo XXI para así tener un referente jurídico de corte histórico. Se han diferenciado estas leyes en tres específicos grupos (Tabla 1):

- El ámbito penal, conteniendo esta área las codificaciones que criminalizan la violencia contra la mujer.

- Las órdenes de protección, entendidas como las medidas que directamente contemplaron los legisladores para hacer efectiva los derechos de las mujeres víctimas de violencia.

- Un ámbito referente al ámbito social y laboral donde se ordena la creación de órganos que den efectivo seguimiento a la situación de violencia contra la mujer de estos dos países en los que se realizó este estudio.

Tabla 1

Marco jurídico en Colombia y España respecto la violencia de género

\begin{tabular}{|c|c|}
\hline Colombia & España \\
\hline \multicolumn{2}{|c|}{ Ámbito penal } \\
\hline $\begin{array}{l}\text { Se modifica parcialmente la Ley } 294 \text { de } 1996 \text { y } \\
\text { se reglamenta lo relacionado con la violencia } \\
\text { intrafamiliar (Ley 599, 2000). }\end{array}$ & $\begin{array}{l}\text { Se contempla en el código penal nuevos tipos } \\
\text { penales de violencia contra la mujer (Ley } \\
\text { Orgánica 10, 1995). }\end{array}$ \\
\hline
\end{tabular}




\begin{tabular}{l|l}
\hline $\begin{array}{l}\text { Se relacionan nuevos delitos contra la libertad, } \\
\text { la integridad y la formación sexual y contra la } \\
\text { familia, entre otros (Ley 640, 2001). }\end{array}$ & $\begin{array}{l}\text { Se expide ley que toma medidas concretas en } \\
\text { materia de seguridad ciudadana, violencia } \\
\text { doméstica e integración social de los extranjeros } \\
\text { (Ley Orgánica 11, 2003). }\end{array}$ \\
\hline $\begin{array}{l}\text { La conciliación judicial y extrajudicial y dispone } \\
\text { medidas de protección cuando se trata de } \\
\text { problemas en que se encuentran miembros de la } \\
\text { familia (Ley 640, 2001). Nuevas normas para la } \\
\text { prevención y la sanción de la violencia } \\
\text { intrafamiliar (Decreto 652, 2001). }\end{array}$ & $\begin{array}{l}\text { Toma medidas de protección integral contra la } \\
\text { lolencia de género y coordinando, en definitiva, } \\
\text { que pretende ser integral y óptima en su } \\
\text { funcionamiento (Ley Orgánica 1, 2004). }\end{array}$ \\
\hline $\begin{array}{l}\text { Exclusión de la obligatoriedad de la asistencia de } \\
\text { las mujeres a los planes de intervención familiar } \\
\text { cuando es víctima de violencia por su pareja (Ley } \\
\text { 1361, 2009). }\end{array}$ & $\begin{array}{l}\text { Se expide el estatuto de la víctima del delito (Ley } \\
\text { las Víctimas del Delito (Real Decreto 1109, 2015). }\end{array}$ \\
\hline $\begin{array}{l}\text { Tipificación del delito de feminicidio cómo un } \\
\text { delito autónomo más grave que el homicidio. } \\
\text { (Ley 1761, 2015). }\end{array}$ & \\
\hline
\end{tabular}

Órdenes de protección

Se dictan normas para prevenir remediar y sancionar la violencia Intrafamiliar (Ley 294, 1996).

Normas de sensibilización, prevención y sanción de formas de violencia y discriminación contra las mujeres. Reformas legislativas (Ley 1257, 2008).

Tipificación del delito de feminicidio cómo un delito autónomo más grave que el homicidio. (Ley 1761, 2015).
Se expide la normativa de protección de las víctimas de la violencia doméstica (Ley 27,2003 ).

Se expide la ley de reconocimiento mutuo de resoluciones penales en la Unión Europea (Ley 23, 2014).

Se aprueban los modelos de remisión al Registro Central para la Protección de las Víctimas de Violencia Domestica de la información (Orden JUS 242, 2009).

\begin{tabular}{l|l}
\hline \multicolumn{2}{c}{ Ámbito social y laboral } \\
\hline $\begin{array}{l}\text { Constitución del Observatorio de Género (Ley } \\
882,2004) .\end{array}$ & $\begin{array}{l}\text { Se aprueba el programa de inserción socio- } \\
\text { laboral para mujeres víctimas de violencia de } \\
\text { género (Real Decreto 1917, 2008). }\end{array}$ \\
\hline $\begin{array}{l}\text { Legislación contra la violencia en el trabajo y el } \\
\text { acoso laboral. Debe tenerse en cuenta que la } \\
\text { principal víctima en términos de estadísticas es } \\
\text { la mujer (Ley 1023, 2006). }\end{array}$ & $\begin{array}{l}\text { Se establecen las funciones, el régimen de } \\
\text { Ouncionamiento y la composición del } \\
\text { (Real Decreto 253, 2006). }\end{array}$ \\
\hline $\begin{array}{l}\text { Creación del comité interinstitucional consultivo } \\
\text { para la prevención de la violencia sexual y la } \\
\text { atención integral de los niños, las niñas y los } \\
\text { adolescentes abusados sexualmente (Ley 1232, }\end{array}$ & \\
\hline IQUAL. REVISTA DE GÉNERO E IGUALDAD, 2022, 5, 79-97 \\
ISSN. 2603-851X \\
DOI. http://dx.doi.org/10.6018/iqual.479851
\end{tabular}


2008).

Fuente: Moreno, et al. (2019).

\section{Metodología}

El diseño utilizado en esta investigación cuantitativa de tipo descriptivo-correlacional fue de corte exploratorio; habiendo participado 106 mujeres usuarias del servicio Centro de Información de la Mujer (CIM) del municipio de Coirós (Galicia, España) y del Centro de Orientación de la Mujer (COM) de Cartagena de Indias (Bolívar, Colombia)4. Los criterios de inclusión fueron ser mujer mayor de edad que haya asistido a uno de estos dos lugares durante el año 2019 y ser reconocidas por los/as trabajadores/as del CIM o del COM como mujeres maltratadas después de la realización de una entrevista de diagnóstico, abriéndoles de este modo un expediente personalizado para realizar el pertinente seguimiento de su caso.

La selección de los dos centros de atención a víctimas de la violencia de género con los que se ha trabajado se justificaría en función a pertenecer ambas instituciones a las zonas geográficas de influencia del autor principal del presente documento (Galicia y Bolívar). Previamente a nuestro estudio ya se habian identificado a muchas usuarias del COM como actuales residentes de Cartagena de Indias que en el pasado fueron desplazadas de sus pueblos de origen por la violencia generalizada en el departamento de Bolívar, queriéndose hacer una comparativa lo más exacta posible entre personas provenientes del ámbito rural con el CIM de Coirós (un municipio rural próximo a La Coruña), ya que este tipo de servicios atencionales a víctimas de violencia de género están ausentes en el rural colombiano. Gracias a la aceptación e interés que mostraron las autoridades responsables del CIM (Ayuntamiento de Coirós) y el COM (Corporación Universitaria Rafael Núñez) se pudo llevar a cabo la fase inicial de un macroproyecto de investigación por el cual se pretende comparar en un futuro este tipo de servicios en España y Colombia a nivel nacional.

Los objetivos de la investigación se han centrado principalmente en solventar tres preguntas problema. 1. ¿Cuáles son los patrones prototípicos de las personas que acuden al CIM y al COM además de averiguar si esas características son comunes o no en ambos espacios? 2. ¿Las mujeres maltratadas conocían las funciones de este tipo de servicios

\footnotetext{
4 El Centro de Información a la Mujer (CIM) es un servicio gratuito del ayuntamiento de Coirós que nació en el año 1995, siendo uno de los primeros centros de este tipo en Galicia, brindando ayuda profesional de tipo psicológico, jurídico y de igualdad. De igual manera el Centro de Orientación a la Mujer (COM) hace parte de un convenio interinstitucional entre la Alcaldía Mayor de Cartagena de Indias y la Corporación Universitaria Rafael Núñez, el cual inició sus actividades en el año 2016, convirtiéndose así en el primer centro de orientación a la mujer de esta ciudad colombiana.
}

IQUAL. REVISTA DE GÉNERO E IGUALDAD, 2022, 5, 79-97 ISSN. 2603-851X DOI. http://dx.doi.org/10.6018/iqual.479851 
atencionales? 3. ¿Habrá diferencias significativas en el nivel de satisfacción global y particular del CIM y del COM en base a las altas discrepancias de las tasas de feminicidios de las dos regiones geográficas analizadas? Las hipótesis de investigación manejadas fueron las siguientes:

- $\mathrm{H}_{1}{ }_{0}=$ No habrá diferencias significativas en la edad, el número de hijos y personas mayores dependientes a su cargo, el tipo de profesión, el estado civil o el tipo de ayuda que demandan las mujeres que son atendidas en el CIM y el COM.

- $\quad \mathrm{H}_{2}=$ No habrá diferencias significativas en el conocimiento previo de las usuarias respecto las funciones del CIM y del COM.

- $\quad \mathrm{H}_{30}=$ No habrá diferencias significativas en los niveles de satisfacción general y de cada área específica de las mujeres que son atendidas en el CIM y el COM.

Se recogieron informaciones de mujeres que acudieron al CIM y que recibieron ayuda del COM utilizándose un cuestionario de satisfacción, elaborado ad hoc por los propios investigadores (adaptado éste a cada contexto específico) basándonos en los principios reguladores de la histórica prueba estandarizada para la medición de la calidad de servicios SERVQUAL (Parasuraman et al. 1988). El material quedó compuesto por diferentes apartados en el que se les solicitaba a la usuaria datos sobre la satisfacción general por el trato recibido y de las distintas áreas de los centros que había requerido (psicológica, jurídica, etc.).

Se realizó un análisis descriptivo con las variables socio-demográficas (cuartiles de edad, profesión, número de hijos...) para identificar las características personales de los dos subgrupos (españolas y colombianas), además de detectar las diferencias entre los niveles de satisfacción general y específica de todas las mujeres. Para ello, utilizando el software estadístico SPSS (v. 23) se manejaron pruebas no paramétricas (U de MannWhitney y Kruskal-Wallis) debido a que no se cumplió el supuesto de normalidad en la variable criterio principal (satisfacción general).

Todas las usuarias participaron de forma voluntaria y anónima cubriendo los cuestionarios en los propios centros, habiendo firmado previamente un consentimiento informado en el que se explicaba la motivación de la investigación y su finalidad: identificar diferencias en el perfil de las mujeres y niveles de satisfacción en víctimas de malos tratos atendidas por estos espacios, en un entorno del campo gallego de un país desarrollado (Coirós, Galicia) y las de una urbe de un país en vías de desarrollo con una gran parte de sus residentes provenientes del entorno rural cercano (Cartagena, Bolívar).

IQUAL. REVISTA DE GÉNERO E IGUALDAD, 2022, 5, 79-97

ISSN. 2603-851X

DOI. http://dx.doi.org/10.6018/iqual. 479851 
Como principales limitantes del estudio que se presenta en estas páginas se debe señalar el tamaño limitado de las poblaciones (prácticamente un centenar de personas) y la reducida potencia estadística asociada a las pruebas no paramétricas manejadas. Se recuerda el carácter exploratorio de esta investigación, esperando en un futuro próximo poder ampliar los datos de las dos regiones geográficas con una mayor cantidad de mujeres encuestadas procedentes de diferentes centros de atención a víctimas de violencia de género de España y Colombia.

\section{Resultados}

La muestra de usuarias final de ambos organismos fueron de un total de 106 mujeres, 63 personas en el CIM y 43 en el COM. Respecto el alcance geográfico de los centros, es relevante indicar que al CIM, a pesar de estar ubicado en una zona rural escasamente poblada, habían acudido personas procedentes de pueblos de tamaño medio de diez municipios distintos (de los cuales, algunos de ellos contaban con instalaciones similares con la que se ha trabajado) y ningún sujeto provenía de la capital de provincia (La Coruña) ${ }^{5}$. Este número fue mayor al de las mujeres que ha atendido el COM aun cuando éste se encuentra bien posicionado en una gran ciudad, capital de departamento. De este modo, el alcance geográfico del COM fue más limitado que el del CIM siendo la mayoría de mujeres atendidas residentes de Cartagena (pero no originarias de la ciudad), pues solamente dos (2) usuarias del COM eran de municipios rurales de las cercanías (Turbaco y Arjona).

Los datos hallados han determinado unas características de los perfiles de las usuarias diferentes en función de su origen geográfico. Por lo tanto, se rechazaría la primera hipótesis nula que se ha planteado en esta investigación, siendo la única característica común el estado civil. De manera más detallada hemos podido especificar los siguientes patrones de usuarias atendidas por el CIM y el COM:

- $\quad$ Usuaria del CIM: mujer de mediana edad ( $M=45.11$ años) con un índice de natalidad de 1.65 hijos/mujer, trabajadora por cuenta ajena en el sector servicios, predominantemente soltera y sin personas mayores a su cargo. Los servicios que más demandó fueron los relacionados con la ayuda psicológica.

5 Destacando la procedencia de mujeres de municipios relevantes de la comarca de As Mariñas como Betanzos y Miño. 
- Usuaria del COM: mujer joven ( $\quad 333.33$ años) con un índice de natalidad de 2.33 hijos/mujer, mayoritariamente ama de casa o desempleada, siendo su estado civil el de soltera y con personas mayores a su cargo. Las demandantes habían acudido por ayuda en todas las áreas de intervención que tiene esta institución.

Vista esta comparativa se ha evidenciado que la mujer maltratada atendida por el COM presentó una mayor indefensión que su par gallega. Ésta se encontraba en una mayor situación de vulnerabilidad, además de los factores ambientales mencionados al inicio del documento, por ser más dependiente económicamente al no tener ingresos propios y tener a su cargo a un mayor número de menores de edad. Con relación a las personas mayores que las mujeres tenían a su cargo, esta situación predominó más en Colombia al convivir las víctimas con sus progenitores, evidenciándose así que en muchas ocasiones el soporte financiero de las víctimas de malos tratos en Cartagena eran sus familiares directos, en contraste con la situación de Galicia donde la víctima sería el sostén principal de su propio hogar.

Sobre la segunda hipótesis de trabajo, tanto las mujeres que fueron atendidas por el CIM como por el COM, estas personas ignoraban en su gran mayoría las funciones específicas de estos espacios. En ambas entidades las tasas de desconocimiento de estos servicios fueron superiores al $70 \%$ de la muestra total, no habiendo diferencias significativas al respecto y por ello habiéndose aceptado la segunda hipótesis nula. Un aspecto que evocaría una carencia que debe ser suplida para así llegar a una mayor población vulnerable en estas dos regiones.

En relación con la satisfacción general y específica de las usuarias sobre los servicios que ofrecieron estos dos centros de atención a las mujeres maltratadas (Tabla 2), debimos aceptar la tercera hipótesis nula propuesta. En ambas instituciones se obtuvieron notas extremadamente positivas ( $\mathrm{CIM}=4.90 / \mathrm{COM}=4.88$ ), prácticamente rozándose la máxima nota posible (5.00); evidenciándose así el apoyo de las víctimas gallegas y bolivarenses ante la posibilidad de acceder a este tipo de servicios de carácter gratuito y confidencial.

Tabla 2

Nivel de satisfacción de las usuarias en los centros de atención

\begin{tabular}{lcccc}
\hline & $\begin{array}{c}\text { Colombia } \\
\text { (No Sujetos) }\end{array}$ & $\begin{array}{c}\text { España (Nº } \\
\text { Sujetos) }\end{array}$ & $\begin{array}{c}\text { Colombia } \\
\text { (Puntuación) }\end{array}$ & $\begin{array}{c}\text { España } \\
\text { (Puntuación) }\end{array}$ \\
\hline Satisfacción general & 43 & 62 & 4.88 & 4.90 \\
Satisfacción jurídica & 43 & 37 & 4.77 & 4.89 \\
Satisfacción psicológica & 38 & 54 & 4.87 & 4.94 \\
\hline
\end{tabular}

IQUAL. REVISTA DE GÉNERO E IGUALDAD, 2022, 5, 79-97

ISSN. 2603-851X

DOI. http://dx.doi.org/10.6018/iqual.479851 


\begin{tabular}{lllll}
\hline Satisfacción igualdad & 43 & 33 & 4.91 & 4.91 \\
Satisfacción informativa & 43 & 45 & 4.86 & 4.93 \\
\hline
\end{tabular}

Fuente: Elaboración propia (2019).

Aunque las puntuaciones de satisfacción global y específicas en función de su origen geográfico no fueron significativas o incluso otras variables no planteadas en la investigación tampoco reflejaron variaciones relevantes en la satisfacción general a nivel matemático, tales como los cuartiles de edad, o sí el sujeto conocía previamente (o no) la existencia de los espacios analizados ( $p \geq .05$ ); al analizar los datos de manera más exhaustiva, especificando más a las usuarias por subgrupos y no por el origen geográfico (cuartiles de edad / estado civil - satisfacciones específicas) sí se encontraron diferencias importantes que se requiere mencionar brevemente.

Se encontraron diferencias relevantes en satisfacción de servicios específicos de los dos centros entre la edad y la satisfacción del área jurídica $(p=.013)$, siendo las personas de menor edad las que aprecian más la labor de las abogadas que trabajan en el CIM y en el COM y, de forma llamativa las mujeres cuyo estado civil eran solteras puntuaron mejor que sus otras pares en los campos de profesionalidad $(p=.037)$ y atención de los trabajadores de los centros $(p=.037)$. Dato interesante al recordar que las puntuaciones de satisfacción más bajas, tanto en Coirós como en Cartagena, fueron los derivados de la atención jurídica.

\section{Discusión de resultados y conclusiones}

La Asociación de Psicología Americana (APA) (s.f) estimaba que el riesgo de ser víctima de violencia de género se incrementa al tener bajos recursos, poseer un bajo nivel educativo, ser adolescente, y ser dependiente de sustancias psicoactivas. Estos datos reflejarían a la perfección el perfil de usuaria del COM, asociándose a esta situación muchas otras problemáticas sociales que sufre el departamento de Bolívar, la ciudad de Cartagena de Indias o el Caribe en general, tal como el elevado desempleo femenino o los embarazos a temprana edad (Watson, 2018), siendo las mujeres solteras y más jóvenes las más expuestas a ser víctimas de violencia de género (González y Garrido, 2015; Watson, 2018; OMS, 2018; 2019). Datos más próximos a los de países africanos (Chernet y Cherie, 2020) que a los encontrados en España (Ministerio de Igualdad de España, 2021).

La obtención de buenas valoraciones en satisfacción general tanto de las usuarias del CIM y del COM fueron coincidentes con los datos obtenidos por González y Garrido (2015) en España, al identificar que el $80 \%$ de las mujeres víctimas de violencia de género expresaron estar muy satisfechas por la atención recibida por las autoridades policiales de 
esa nación europea, no habiendo estudios similares en el entorno del Caribe colombiano. Un aspecto entonces desconocido hasta el momento y que esperamos que, con esta pequeña aportación científica, se permita dilucidar en parte.

Se asumiría entonces, con extrema cautela debido al carácter exploratorio de esta investigación, que la cantidad de recursos humanos y económicos ofrecidos a las mujeres maltratadas por estos organismos no es el factor que determina la insatisfacción de las usuarias, ya que lógicamente el CIM ha manejado un mayor portafolio de actividades y servicios por ser esta institución procedente de un país desarrollado frente al COM. Un punto clave el que se acaba de comentar que permitiría profundizar una línea de investigación futura en la que, además de ampliar la medición en España y Colombia como se había indicado en párrafos anteriores, también se podría comenzar la observación de la actitud hacia la violencia de género de los profesionales de este tipo de centros ( $u$ otros similares) como hicieron Rojas-Loria et al. (2015) pero en un nuevo contexto como el de Colombia.

En conclusión, la problemática de género, y particularmente la violencia de género hacia las mujeres, representa una situación de interés para la sociedad en general y para los entes públicos y privados encargados de promover la prevención y erradicación de la misma. Como fruto de esto, se ha dado la creación de centros de atención especializados en dicha problemática cuya demanda aumenta cada día, si bien el desconocimiento de estas instituciones es considerable como se ha visto en este artículo científico. Por lo anterior, el mayor reto que enfrentan los centros asistenciales no tiene que ver solo con el impacto, la cobertura y la accesibilidad. Entran otras dos variables en juego que son la satisfacción de las mismas usuarias (y el reto de mantener esos elevados puntajes de opinión) y la visibilidad de este tipo de servicios atencionales a mujeres maltratadas que es más que mejorable.

Por último, el ser conscientes de que la violencia de género es una problemática de carácter público y de interés para la sociedad colombiana y española en general, nos permite entender que a través de ésta se incurre en la violación de derechos fundamentales inherentes a las mujeres. El realizar este tipo de investigaciones comparativas en regiones de Colombia como Bolívar y buscar posibles modelos a seguir como el que maneja el CIM de Coirós, resultaría beneficioso para una sociedad que sufre la lacra de la violencia y que desgraciadamente acepta la violencia contra la mujer como algo normal (Calderón et al., 2013; Zakzuk y Vázquez-Miraz, 2021). Es por ello por lo que al caracterizar a las poblaciones más vulnerables se podrían poner en marcha mecanismos de prevención y eliminación de las actitudes, pensamientos y comportamientos que perpetúan la desigualdad entre hombres y mujeres más efectivos,

IQUAL. REVISTA DE GÉNERO E IGUALDAD, 2022, 5, 79-97

ISSN. 2603-851X

DOI. http://dx.doi.org/10.6018/iqual.479851 
en aras de alcanzar el anhelado objetivo final que es la erradicación de la violencia de género.

\section{Bibliografía}

Asociación de Psicología Americana (APA) (s.f). Violencia contra de la pareja. Recuperado de: https://www.apa.org/topics/violence/violencia-pareja

Beauvoir, S. (1949). El segundo sexo. Madrid, España: Cátedra.

Bem, S.L. (1974). The measurement of psychological androgyny. Journal of Consulting and Clinical Psychology, 42(2), 165-172. doi: http://dx.doi.org/10.1037/hoo36215

Bernal, G. (2011). Visibilizar la violencia de género. Sistematización de la experiencia en género. Bogotá, Colombia: Agencia Alemana para la Cooperación Internacional.

Calderón, M.C., Gómez, C. y Murad, R. (2013). Historias de violencia, roles, prácticas y discursos legitimadores. Violencia contra las mujeres en Colombia 2000-2010. Bogotá, Colombia: Ministerio de Salud de Colombia.

Chernet, A. G., y Cherie, K. T. (2020). Prevalence of intimate partner violence against women and associated factors in Ethiopia. BMC women's health, 20(1).doi: https://doi.org/10.1186/s12905-020-0892-1

Congreso de Colombia (1996). Ley 294 de 16 de julio de 1996. DO: 42.836

- (2000). Ley 599 de 24 de julio. DO: 44.097

- (2001). Ley 640 de 05 de enero. DO: 44.303

- (2004). Ley 882 de 02 de junio. DO: 45.568

- (2005). Ley 984 de 12 de agosto. DO: 46.002

- (2006). Ley 1023 de 03 de mayo. DO: 46.259

- (2008). Ley 1232 de 17 de julio. DO: 47.053

- (2008). Ley 1257 de 04 de diciembre. DO: 47.913

- (2009). Ley 1361 de 03 de diciembre. DO: 47.552

- (2015). Ley 1761 de o6 de julio. DO: 49.565

Fernández-Ruiz, J.M. (2019). La Ley de Violencia Intrafamiliar, el bien jurídico protegido y el patriarcado: un estudio preliminar.Política Criminal: Revista Electrónica Semestral de Políticas Públicas en Materias Penales, 14(28).

Gámez-Guadix, M., Borrajo, E. y Calvete, E. (2018). Partner abuse, control and violence through internet and smartphones : Characteristics, evaluation and prevention. Papeles del Psicólogo, 39(3), 218-227. doi: https://doi.org/10.23923/pap.psicol2018.2874 
Gobierno de España (1995). Ley Orgánica 10 / 1995, de 23 de noviembre, del Código Penal. Boletín Oficial del Estado, 281.

- (2003). Ley 27/2003, de 31 de julio, reguladora de la Orden de protección de las víctimas de la violencia doméstica. Boletín Oficial del Estado, 183.

- (2003). Ley Orgánica 11/2003, de 29 de septiembre, de medidas concretas en materia de seguridad ciudadana, violencia doméstica e integración social de los extranjeros. Boletín Oficial del Estado, 234.

- (2004). Ley 1/2004, de 28 de diciembre, de Medidas de Protección Integral Contra la Violencia de Género. Boletín Oficial del Estado, 313.

- (2006). Real Decreto 253/2006, de 3 de marzo, por el que se establecen las funciones, el régimen de funcionamiento y la composición del Observatorio Estatal de Violencia sobre la Mujer, y se modifica el Real Decreto 1600/2004, de 2 de julio, por el que se desarrolla la estructura orgánica básica del Ministerio de Trabajo y Asuntos Sociales. Boletín Oficial del Estado, 62.

- (2008). Real Decreto 1917/2008, de 21 de noviembre, por el que se aprueba el programa de inserción sociolaboral para mujeres víctimas de violencia de género. Boletín Oficial del Estado, 297.

- (2009). Real Decreto 95/2009, de 6 de febrero, por el que se regula el Sistema de registros administrativos de apoyo a la Administración de Justicia. Boletín Oficial del Estado, 33

- (2009). Orden JUS/242/2009, de 10 de febrero, por la que se aprueban los modelos de remisión al Registro Central para la Protección de las Víctimas de Violencia Doméstica de la información que debe inscribirse en el mismo. Boletín Oficial del Estado, 39.

- (2014). Ley 23/2014, de 20 de noviembre, de reconocimiento mutuo de resoluciones penales en la Unión Europea. Boletín Oficial del Estado, 282.

- (2015). Orden JUS/375/2015, de 26 de febrero, por la que se modifica la Orden JUS/242/2009, de 10 de febrero, por la que se aprueban los modelos de remisión al Registro Central para la Protección de las Víctimas de Violencia Doméstica de la información que debe inscribirse en el mismo. Boletín Oficial del Estado, 56.

- (2015). Ley Orgánica 4 / 2015, de 11 de diciembre, por el que se desarrolla la Ley 4/2015, de 27 de abril, del Estatuto de la víctima del delito, y se regulan las Oficinas de Asistencia a las Víctimas del Delito. Boletín Oficial del Estado, 77.

- (2015). Real Decreto 1109 / 2015, de 9 de 30 de marzo de protección de la seguridad ciudadana. Boletín Oficial del Estado, 312.

González, J.L., y Garrido, M.J. (2015). Satisfacción de las víctimas de violencia de género con la actuación policial en España. Validación del Sistema VioGen. Anuario de Psicología Jurídica, 25(1), 29-38. doi: http://dx.doi.org/10.1016/j.apj.2015.02.003

IQUAL. REVISTA DE GÉNERO E IGUALDAD, 2022, 5, 79-97

ISSN. 2603-851X

DOI. http://dx.doi.org/10.6o18/iqual.479851 
López, E. (2004). La figura del agresor en la violencia de género: características personales e intervención. Papeles del psicólogo, 25(88), 31-38.

Ministerio de Igualdad de España (Ed.) (2021). Ficha estadística de víctimas mortales por Violencia de Género. Año 2021. Madrid. Recuperado de: https://violenciagenero.igualdad.gob.es/violenciaEnCifras/victimasMortales/ficha Mujeres/pdf/VMortales $2021 \quad 1108$ 1.pdf

Ministerio de Salud de Colombia (Ed.) (1999). Guía de atención de la mujer maltratada. Bogotá, Colombia.

Ministerio de Presidencia de España (Ed.) (2019). Estadística de Víctimas Mortales por Violencia de Género. Recuperado de: https://violenciagenero.igualdad.gob.es/violenciaEnCifras/victimasMortales/ficha Mujeres/mortales CCAA/datos 2018/docs/Galicia_2018.pdf

Ministerio de Tecnologías de la Información y las Comunicaciones de Colombia (Ed.) (2021). Feminicidios en Colombia (2015-2020). Bogotá. Recuperado de: https://www.datos.gov.co/Justicia-y-Derecho/FEMINICIDIOS-EN-COLOMBIA2015-2020-/besk-ypw5

Moreno, F., Carmona, J., Ocampo, D., Vargas, A., y Alvarado, S. (2019). Campañas de prevención de violencia de género en Colombia y España: un análisis desde la psicología social y la publicidad social. Manizales, Colombia: Universidad de Manizales.

Munévar, D. y Mena, L. (2009). Violencia estructural de género. Opiniones, debates y controversias, 59, 356-365.

ONU Mujeres (2012). Historia y origen de los refugios para la mujer. Recuperado de: http://www.endvawnow.org/es/articles/1368-historia-y-origen-de-

Organización Mundial de la Salud (OMS) (Ed.) (1998). Violencia contra la mujer. Recuperado de: https://www.who.int/gender/violence/violencia infopack1.pdf

- (2019). Preventing violence against women. Ginebra.

- (2018). Estudio multipaís de la OMS sobre salud de la mujer y violencia doméstica contra la mujer: primeros resultados sobre prevalencia, eventos relativos a la salud y respuestas de las mujeres a dicha violencia. Ginebra.

Parasuraman, A., Berry, L. y Zeithaml, V.A. (1988). SERVQUAL: A Multiple-Item Scale for Measuring Customer Perceptions of Service Quality. Journal of Retailing, 64, 1240.

Presidencia de la Republica de Colombia (2001). Decreto 652 de 16 de abril. DO: 44.394

Rojas-Loría, K., Gutiérrez-Rosado, T., Alvarado, R. y Fernández-Sánchez, A. (2015). Actitud hacia la violencia de género de los profesionales de Atención 
Primaria: estudio comparativo entre Cataluña y Costa Rica. Atención Primaria, 47(8), 490-497. doi: https://doi.org/10.1016/j.aprim.2014.10.008

Romero, I. (2010). Intervención en violencia de género: consideraciones en torno al tratamiento. Psychosocial intervention, 19(2), 191-199.

Sahagún, M y Arias, J. (2018). Violencia de género desde un abordaje interdisciplinar. Bogotá: Universidad Sergio Arboleda y Corporación Universitaria del Caribe.

San Martín, A. (2012). Violencia de género y cultura (Tesis doctoral). Universidad de La Coruña, La Coruña, España. Recuperado de: https://ruc.udc.es/dspace/handle/2183/10025

Vázquez-Miraz, P. (2017). Violencia machista y menores: efectos en los niños a causa del tipo de educación parental recibida (Tesis doctoral), Universidad de La Coruña, A. Coruña, España. Recuperado de: https://ruc.udc.es/dspace/handle/2183/19541

Watson, C. (2018). Women's Health Survey 2016. ONU Mujeres.

Yugueros, A.J. (2014). La violencia contra las mujeres: conceptos y causas. Barataria.

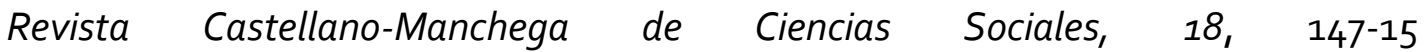

- (2021). Violencia de género, seguridad de las víctimas desde la perspectiva psicosocial. iQUAL. Revista de Género e Igualdad, 4, 6o-88. doi: https://doi.org/10.6018/iqual.435621

Zakzuk-López, S. y Vázquez-Miraz, P. (2021). Aceptación de mitos sobre agresión sexual en universitarios colombianos. Cuestiones de género: de la igualdad y la diferencia, 16, 775-79o. doi: http://dx.doi.org/10.18002/cg.voi16.6903

Cómo referenciar este artículo/How to reference this article $(*):$

Vázquez-Miraz, P., Quintana, A., y Riveira, B. (2022). Atención en centros asistenciales para la mujer víctima de violencia de género de Coirós (Galicia) y Cartagena de Indias: un análisis comparado. iQUAL. Revista de Género e Igualdad, 5, 79-97, doi: 10.6018/iqual.479851

Vázquez-Miraz, P., Quintana, A., y Riveira, B. (2022). Atención en centros asistenciales para la mujer víctima de violencia de género de Coirós (Galicia) y Cartagena de Indias: un análisis comparado. [Attention in healthcare centers for women victims of gender violence of Coirós (Galicia) and Cartagena de Indias: a comparative analysis]. iQUAL. Revista de Género e Igualdad, 5, 79-97, doi: 10.6018/iqual.479851

(*) La autoría colectiva responde a una contribución conjunta en todos los apartados.

IQUAL. REVISTA DE GÉNERO E IGUALDAD, 2022, 5, 79-97

ISSN. 2603-851X

DOI. http://dx.doi.org/10.6o18/iqual.479851 


\section{Anexos}

Anexo 1. Cuestionario del Centro de Informacion a la Mujer (CIM)
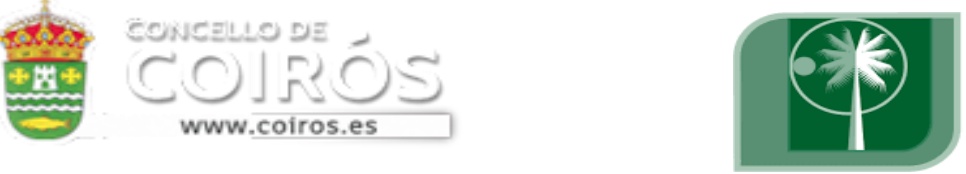

Universidad Tecnológica de Bolívar CARTAGENADEINDIAS

\section{CUESTIONARIO SOBRE SATISFACCIÓN DOS SERVIZOS DO CIM (Centro de Información á Muller de Coirós)}

Data:

\begin{tabular}{|l|l|}
\hline Idade & \\
\hline Concello de residencia & \\
\hline Profesión actual & \\
\hline Estado civil & \\
\hline Número de fillas e fillos & \\
\hline Número de persoas dependentes (menores e maiores) ao seu coidado & \\
\hline
\end{tabular}

1. Antes de acudir ao CIM, tiñas claro a utilidade deste centro e a súa función? [Marca cun X]

SI:

NON:

2. Valora [cunha X] os servizos que usaches do CIM acordo coa túa satisfacción seguinte baremo (se algún dos servizos non foron requeridos, deixa o apartado en branco):

\begin{tabular}{|l|c|c|c|c|c|}
\hline \multicolumn{1}{|c|}{ Área de traballo } & $\begin{array}{c}1 \\
\text { Moi mal }\end{array}$ & $\begin{array}{c}2 \\
\text { Mal }\end{array}$ & $\begin{array}{c}3 \\
\text { Indiferente }\end{array}$ & $\begin{array}{c}4 \\
\text { Ben }\end{array}$ & $\begin{array}{c}5 \\
\text { Moi ben }\end{array}$ \\
\hline A. Xurídica & 1 & 2 & 3 & 4 & 5 \\
\hline A. Psicolóxica & 1 & 2 & 3 & 4 & 5 \\
\hline A. Igualdade & 1 & 2 & 3 & 4 & 5 \\
\hline $\begin{array}{l}\text { A. Informativa, } \\
\text { axudas e recursos }\end{array}$ & 1 & 2 & 3 & 4 & 5 \\
\hline
\end{tabular}

3. Puntúa tamén os seguintes aspectos do CIM acordo co mesmo baremo:

\begin{tabular}{|l|c|c|c|c|c|}
\hline & $\begin{array}{c}1 \\
\text { Moi mal }\end{array}$ & $\begin{array}{c}2 \\
\text { Mal }\end{array}$ & $\begin{array}{c}3 \\
\text { Indiferente }\end{array}$ & $\begin{array}{c}4 \\
\text { Ben }\end{array}$ & $\begin{array}{c}5 \\
\text { Moi ben }\end{array}$ \\
\hline A valía das profesionais do CIM & 1 & 2 & 3 & 4 & 5 \\
\hline As instalacións físicas do departamento & 1 & 2 & 3 & 4 & 5 \\
\hline A organización do centro & 1 & 2 & 3 & 4 & 5 \\
\hline As actividades realizadas polo CIM & 1 & 2 & 3 & 4 & 5 \\
\hline O horario de atención ás persoas usuarias & 1 & 2 & 3 & 4 & 5 \\
\hline A atención prestada das profesionais ao público & 1 & 2 & 3 & 4 & 5 \\
\hline A información transmitida polo Centro & 1 & 2 & 3 & 4 & 5 \\
\hline
\end{tabular}

4. Finalmente, indica cun número (de 1 a 5) a túa valoración xeral do CIM:

Moitas grazas pola túa participación e polo teu tempo

IQUAL. REVISTA DE GÉNERO E IGUALDAD, 2022, 5, 79-97 
Anexo 2. Cuestionario del Centro De Orientacion a la Mujer (COM)

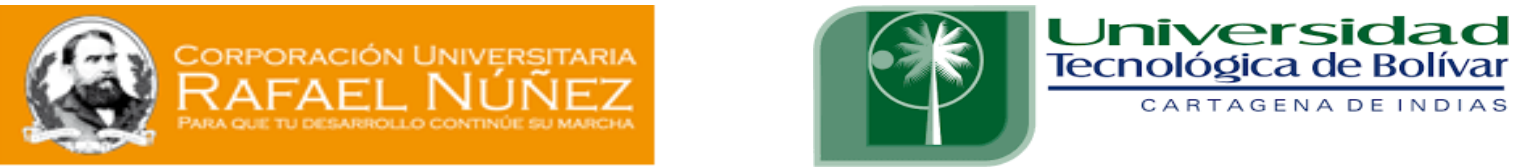

\section{CUESTIONARIO SOBRE SATISFACCIÓN DE LOS SERVICIOS DEL COM (Centro de Orientación a la Mujer de la Corporación Universitaria Rafael Núñez)}

Fecha:

\begin{tabular}{|l|l|}
\hline Edad & \\
\hline Municipio de residencia & \\
\hline Profesión actual & \\
\hline Estado civil & \\
\hline Número de hijas e hijos & \\
\hline Número de personas dependientes (menores y mayores) a su cuidado & \\
\hline
\end{tabular}

5. Antes de acudir al COM, ¿tenías claro la utilidad de este centro y su función? [Marca con un $X]$

SI:

NO:

6. Valora [con un X] los servicios que has usado del COM, de acuerdo con tu satisfacción, respecto el siguiente baremo (si algún servicio no fue requerido, deja el apartado sin contestar):

\begin{tabular}{|l|c|c|c|c|c|}
\hline \multicolumn{1}{|c|}{ Área de trabajo } & $\begin{array}{c}1 \\
\text { Muy mal }\end{array}$ & $\begin{array}{c}2 \\
\text { Mal }\end{array}$ & $\begin{array}{c}3 \\
\text { Indiferente }\end{array}$ & $\begin{array}{c}4 \\
\text { Bien }\end{array}$ & $\begin{array}{c}5 \\
\text { Muy bien }\end{array}$ \\
\hline A. Jurídica & 1 & 2 & 3 & 4 & 5 \\
\hline A. Psicológica & 1 & 2 & 3 & 4 & 5 \\
\hline $\begin{array}{l}\text { A. Igualdad } \\
\text { A. Informativa, } \\
\text { ayudas, recursos }\end{array}$ & 1 & 2 & 3 & 4 & 5 \\
\hline
\end{tabular}

7. Puntúa también los siguientes aspectos del COM de acuerdo con el mismo baremo:

\begin{tabular}{|l|c|c|c|c|c|}
\hline & $\begin{array}{c}1 \\
\text { Muy } \\
m a l\end{array}$ & $\begin{array}{c}2 \\
\text { Mal }\end{array}$ & $\begin{array}{c}3 \\
\text { Indiferent } \\
e\end{array}$ & $\begin{array}{c}4 \\
\text { Bien }\end{array}$ & $\begin{array}{c}5 \\
\text { Muy } \\
\text { bien }\end{array}$ \\
\hline La valía de las profesionales del COM & 1 & 2 & 3 & 4 & 5 \\
\hline Las instalaciones físicas del departamento & 1 & 2 & 3 & 4 & 5 \\
\hline La organización del centro & 1 & 2 & 3 & 4 & 5 \\
\hline Las actividades realizadas por el COM & 1 & 2 & 3 & 4 & 5 \\
\hline El horario de atención a las personas usuarias & 1 & 2 & 3 & 4 & 5 \\
\hline La atención prestada de las profesionales & 1 & 2 & 3 & 4 & 5 \\
\hline La información transmitida por el Centro & 1 & 2 & 3 & 4 & 5 \\
\hline
\end{tabular}

8. Finalmente, indica con un número (de 1 a 5) tu valoración general del COM:

IQUAL. REVISTA DE GÉNERO E IGUALDAD, 2022, 5, 79-97

ISSN. 2603-851X

DOI. http://dx.doi.org/10.6018/iqual. 479851 